\title{
Existence and Non-Existence Results for Nonlocal Elliptic Systems via Sub-Supersolution Method
}

\author{
By \\ G. A. Afrouzi, N. T. Chung and S. Shakeri \\ (University of Mazandaran, Iran, Quang Binh University, Vietnam and \\ Islamic Azad University, Iran)
}

\begin{abstract}
In this paper, using a sub-supersolution argument, we prove an existence result on a positive weak solution for a class of nonlocal elliptic systems in bounded domains.

Key Words and Phrases. Nonlocal elliptic systems, Existence, Non-Existence, Positive solution, Sub-supersolution method.

2010 Mathematics Subject Classification Numbers. 35D05, 35J60.
\end{abstract}

\section{Introduction and Preliminaries}

In this paper, we are interested in a class of nonlinear elliptic systems of the form

$$
\left\{\begin{array}{l}
-A\left(\int_{\Omega}|\nabla u|^{2} d x\right) \Delta u=\lambda f(v) \quad \text { in } \Omega \\
-B\left(\int_{\Omega}|\nabla v|^{2} d x\right) \Delta v=\lambda g(u) \quad \text { in } \Omega \\
u=v=0, \quad \text { on } \partial \Omega
\end{array}\right.
$$

where $\Omega \subset \boldsymbol{R}^{N}(N \geq 3)$ is a bounded domain with smooth boundary $\partial \Omega$, $A, B: \boldsymbol{R}^{+} \rightarrow \boldsymbol{R}^{+}$are continuous functions, $1<p<N$ and $\lambda$ is a positive parameter.

Since the first equation in (1.1) contains an integral over $\Omega$, it is no longer a pointwise identity; therefore it is often called a nonlocal problem. This problem models several physical and biological systems, where $u$ describes a process which depends on the average of itself, such as the population density, see [3]. Moreover, problem (1.1) is related to the stationary version of the Kirchhoff equation

$$
\rho \frac{\partial^{2} u}{\partial t^{2}}-\left(\frac{P_{0}}{h}+\frac{E}{2 L} \int_{0}^{L}\left|\frac{\partial u}{\partial x}\right|^{2} d x\right) \frac{\partial^{2} u}{\partial x^{2}}=0
$$


presented by Kirchhoff in 1883, see [8]. This equation is an extension of the classical d'Alembert's wave equation by considering the effects of the changes in the length of the string during the vibrations. The parameters in (1.2) have the following meanings: $L$ is the length of the string, $h$ is the area of the crosssection, $E$ is the Young modulus of thematerial, $\rho$ is themass density, and $P_{0}$ is the initial tension.

In recent years, problems involving Kirchhoff type operators have been studied in many papers, we refer to $[1,2,4,5,9,11,12]$, in which the authors have used different methods to get the existence of solutions for (1.1) in the single equation case. In the papers $[10,13]$, Z. Zhang et al. studied the existence of nontrivial sign-changing solutions for this type of problem. In this paper, we are interested in finding positive solutions for system (1.1) via a subsupersolution method. Our paper is motivated by the recent results in $[1,2$, $6,7]$. In the paper [2, Theorem 2], Azzouz and Bensedik studied the existence of a positive solution for the nonlocal problem of the form

$$
\left\{\begin{array}{l}
-M\left(\int_{\Omega}|\nabla u|^{2} d x\right) \Delta u=|u|^{p-1} u+\lambda f(x) \text { in } \Omega, \\
u=0, \text { on } \partial \Omega
\end{array}\right.
$$

where $\Omega$ is a smooth bounded domain in $\boldsymbol{R}^{N}, N \geq 3$ and $p>1$, i.e. the nonlinear term is superlinear at infinity and $f$ is a sign-changing function. Using the sub-supersolution method combining a comparison principle introduced in [1], the authors established the existence of a positive solution for (1.3) when the parameter $\lambda>0$ is small enough. It was also proved in [2] that problem (1.3) has no positive solution when $\lambda$ large enough. In the present paper, we consider system (1.1) in the case when the nonlinearities are "sublinear" at infinity, see the condition $\left(\mathrm{H}_{3}\right)$. We are inspired by the ideas in the interesting paper [6], in which the authors considered system (1.1) in the case $A(t)=B(t) \equiv 1$. More precisely, under suitable conditions on $f, g$, we shall show that system (1.1) has a positive solution for $\lambda>0$ large enough. Moreover, with particular nonlinearities $f, g$ we show the (1.1) has no positive for $\lambda>0$ small enough. To our best knowledge, this is a new research topic for nonlocal problems, see [7]. In the rest of this section, we recall a comparison principle involving Kirchhoff type operators. The readers may concern its proof in the paper [7].

Theorem 1.1. Assume that $M: \boldsymbol{R}^{+} \rightarrow \boldsymbol{R}^{+}$is a continuous and increasing function satisfying

$$
\lim _{t \rightarrow 0^{+}} M(t)=m_{0}>0 .
$$

where $m_{0}$ is a positive constant. 
Assume that $u, v$ are two non-negative functions such that

$$
\left\{\begin{array}{l}
-M\left(\int_{\Omega}|\nabla u|^{2} d x\right) \Delta u \geq-M\left(\int_{\Omega}|\nabla v|^{2} d x\right) \Delta v \text { in } \Omega \\
u=v=0 \text { on } \partial \Omega .
\end{array}\right.
$$

Then $u \geq v$ a.e. in $\Omega$.

\section{Existence result}

In this section, we shall state and prove the main result of this paper. Let us assume the following assumptions:

$\left(H_{1}\right)$ Assume that $A, B: \boldsymbol{R}^{+} \rightarrow \boldsymbol{R}^{+}$are two continuous and increasing functions and there exists $a_{i}, b_{i}>0, i=1,2$, such that

$$
a_{1} \leq A(t) \leq a_{2}, \quad b_{1} \leq B(t) \leq b_{2} \quad \text { for all } t \in \boldsymbol{R}^{+} ;
$$

$\left(H_{2}\right) \quad f, g$ are continuous on $[0,+\infty), C^{1}$ on $(0,+\infty)$, and increasing functions such that

$$
\lim _{t \rightarrow+\infty} f(t)=\lim _{t \rightarrow+\infty} g(t)=+\infty
$$

$\left(H_{3}\right) \quad$ It holds that

$$
\lim _{t \rightarrow+\infty} \frac{f(K g(t))}{t}=0
$$

for every constant $K>0$.

Theorem 2.1. Assume that the conditions $\left(H_{1}\right)-\left(H_{3}\right)$ hold. Then there exists $\lambda^{*}>0$ such that problem (1.1) has a large positive weak solution $(u, v)$ for $\lambda>\lambda^{*}$.

Define $f(t)=0$ and $g(t)=0$ for all $t<0$. We shall establish Theorem 2.1 by constructing a positive subsolution $(\underline{u}, \underline{v}) \in H_{0}^{1}\left(\Omega, \boldsymbol{R}^{2}\right)$ and a positive supersolution $(\bar{u}, \bar{v}) \in H_{0}^{1}\left(\Omega, \boldsymbol{R}^{2}\right)$ of problem (1.1) such that $\underline{u} \leq \bar{u}$ and $\underline{v} \leq \bar{v}$ in $\Omega$, that is, $\underline{u}, \bar{u}, \underline{v}, \bar{v}$ satisfy

$$
\begin{aligned}
& A\left(\int_{\Omega}|\nabla \underline{u}|^{2} d x\right) \int_{\Omega} \nabla \underline{u} \cdot \nabla \varphi d x \leq \lambda \int_{\Omega} f(\underline{v}) \varphi d x \\
& B\left(\int_{\Omega}|\nabla \underline{v}|^{2} d x\right) \int_{\Omega} \nabla \underline{v} \cdot \nabla \varphi d x \leq \lambda \int_{\Omega} g(\underline{u}) \varphi d x
\end{aligned}
$$

and 


$$
\begin{aligned}
& A\left(\int_{\Omega}|\nabla \bar{u}|^{2} d x\right) \int_{\Omega} \nabla \bar{u} \cdot \nabla \varphi d x \geq \lambda \int_{\Omega} f(\bar{v}) \varphi d x \\
& B\left(\int_{\Omega}|\nabla \bar{v}|^{2} d x\right) \int_{\Omega} \nabla \bar{v} \cdot \nabla \varphi d x \geq \lambda \int_{\Omega} g(\bar{u}) \varphi d x
\end{aligned}
$$

for all $\varphi \in H_{0}^{1}(\Omega)$ with $\varphi \geq 0$.

Proof of Theorem 2.1. Let $\lambda_{1}$ be the first eigenvalue of $-\Delta$ with Dirichlet boundary conditions and $\phi_{1}$ the corresponding positive eigenfunction with $\left\|\phi_{1}\right\|_{\infty}=1$. Let $k_{0}, m_{0}, \delta>0$ such that $f(t), g(t) \geq-k_{0}$ for all $t \in \boldsymbol{R}^{+}$and $\left|\nabla \phi_{1}\right|^{2}-\lambda_{1} \phi_{1}^{2} \geq m_{0}$ on $\bar{\Omega}_{\delta}:=\{x \in \Omega: d(x, \partial \Omega) \leq \delta\}$.

For each $\lambda>0$, let us define $\underline{u}=\left(\lambda k_{0} /\left(2 m_{0} a_{1}\right)\right) \phi_{1}^{2}$ and $\underline{v}=\left(\lambda k_{0} /\left(2 m_{0} b_{1}\right)\right) \phi_{1}^{2}$, where $a_{1}, b_{1}$ are given by the condition $\left(H_{1}\right)$. We shall verify that $(\underline{u}, \underline{v})$ is a subsolution of problem (1.1) for $\lambda$ large enough. Indeed, let $\varphi \in H_{0}^{1}(\Omega)$ with $\varphi \geq 0$ in $\Omega$. By $\left(H_{1}\right)$, a simple calculation shows that

$$
\begin{aligned}
& A\left(\int_{\bar{\Omega}_{\delta}}|\nabla \underline{u}|^{2} d x\right) \int_{\bar{\Omega}_{\delta}} \nabla \underline{u} \cdot \nabla \varphi d x \\
& \quad=A\left(\int_{\bar{\Omega}_{\delta}}|\nabla \underline{u}|^{2} d x\right) \frac{\lambda k_{0}}{m_{0} a_{1}} \int_{\bar{\Omega}_{\delta}} \phi \nabla \phi \cdot \nabla \varphi d x \\
& \quad=\frac{\lambda k_{0}}{m_{0} a_{1}} A\left(\int_{\bar{\Omega}_{\delta}}|\nabla \underline{u}|^{2} d x\right)\left\{\int_{\bar{\Omega}_{\delta}} \nabla \phi \cdot \nabla(\phi \varphi) d x-\int_{\bar{\Omega}_{\delta}}|\nabla \phi|^{2} \varphi d x\right\} \\
& \quad=\frac{\lambda k_{0}}{m_{0} a_{1}} A\left(\int_{\bar{\Omega}_{\delta}}|\nabla \underline{u}|^{2} d x\right) \int_{\bar{\Omega}_{\delta}}\left(\lambda_{1} \phi^{2}-|\nabla \phi|^{2}\right) \varphi d x
\end{aligned}
$$

On $\bar{\Omega}_{\delta}$ we have $|\nabla \phi|^{2}-\lambda_{1} \phi^{2} \geq m_{0}$, which implies by using $\left(H_{1}\right)$ that

$$
\begin{aligned}
A\left(\int_{\bar{\Omega}_{\delta}}|\nabla \underline{u}|^{2} d x\right) \int_{\bar{\Omega}_{\delta}} \nabla \underline{u} \cdot \nabla \varphi d x & \leq \frac{\lambda k_{0}}{m_{0}} \int_{\bar{\Omega}_{\delta}}\left(\lambda_{1} \phi^{2}-|\nabla \phi|^{2}\right) \varphi d x \\
& \leq \lambda \int_{\bar{\Omega}_{\delta}} f(\underline{v}) \varphi d x .
\end{aligned}
$$

Next, on $\Omega \backslash \bar{\Omega}_{\delta}$ we have $\phi \geq r$ for some $r>0$, and therefore by the conditions $\left(H_{1}\right),\left(H_{2}\right)$ and the definition of $\underline{v}$, it follows that

$$
\begin{aligned}
\lambda \int_{\Omega \backslash \bar{\Omega}_{\delta}} f(\underline{v}) \varphi d x & \geq \lambda \frac{k_{0} a_{2}}{m_{0} a_{1}} \lambda_{1} \int_{\Omega \backslash \bar{\Omega}_{\delta}} \varphi d x \\
& \geq \lambda \frac{k_{0}}{m_{0} a_{1}} A\left(\int_{\Omega \backslash \bar{\Omega}_{\delta}}|\nabla \underline{u}|^{2} d x\right) \lambda_{1} \int_{\Omega \backslash \bar{\Omega}_{\delta}} \varphi d x
\end{aligned}
$$




$$
\begin{aligned}
& \geq \lambda \frac{k_{0}}{m_{0} a_{1}} A\left(\int_{\Omega \backslash \bar{\Omega}_{\delta}}|\nabla \underline{u}|^{2} d x\right) \int_{\Omega \backslash \bar{\Omega}_{\delta}}\left(\lambda_{1} \phi^{2}-|\nabla \phi|^{2}\right) \varphi d x \\
& =A\left(\int_{\Omega \backslash \bar{\Omega}_{\delta}}|\nabla \underline{u}|^{2} d x\right) \int_{\Omega \backslash \bar{\Omega}_{\delta}} \nabla \underline{u} \cdot \nabla \varphi d x
\end{aligned}
$$

for $\lambda>0$ large enough.

Relations (2.1) and (2.2) imply that

$$
A\left(\int_{\Omega}|\nabla \underline{u}|^{2} d x\right) \int_{\Omega} \nabla \underline{u} \cdot \nabla \varphi d x \leq \lambda \int_{\Omega} f(\underline{v}) \varphi d x
$$

for $\lambda>0$ large enough and any $\varphi \in H_{0}^{1}(\Omega)$ with $\varphi \geq 0$ in $\Omega$. Similarly,

$$
B\left(\int_{\Omega}|\nabla \underline{v}|^{2} d x\right) \int_{\Omega} \nabla \underline{v} \cdot \nabla \varphi d x \leq \lambda \int_{\Omega} g(\underline{u}) \varphi d x
$$

for $\lambda>0$ large enough and any $\varphi \in H_{0}^{1}(\Omega)$ with $\varphi \geq 0$ in $\Omega$. From (2.3) and (2.4), $(\underline{u}, \underline{v})$ is a subsolution of problem (1.1). Moreover, we have $\underline{u}>0$ and $\underline{v}>0$ in $\Omega, \underline{u} \rightarrow+\infty$ and $\underline{v} \rightarrow+\infty$ as $\lambda \rightarrow+\infty$.

Next, we shall construct a supersolution of problem (1.1). For this purpose, we fix $\lambda>0$ for which the subsolution is ensured. Let $\phi_{0}$ be the solution of the following problem

$$
\left\{\begin{array}{l}
-\Delta \varphi=1 \text { in } \Omega \\
\varphi=0, \quad \text { on } \partial \Omega
\end{array}\right.
$$

Let

$$
\bar{u}:=\frac{C \lambda}{R} \phi_{0}, \quad \bar{v}:=\frac{\lambda}{b_{1}} g(C \lambda) \phi_{0},
$$

where $R=\left\|\phi_{0}\right\|_{\infty}>0$ and $C>0$ is a large positive real number to be chosen later. We shall verify that $(\bar{u}, \bar{v})$ is a supersolution of problem (1.1) for $\lambda>0$ large. To this end, let $\varphi \in H_{0}^{1}(\Omega)$ with $\varphi \geq 0$ in $\Omega$, from $\left(H_{1}\right)$, we have

$$
\begin{aligned}
A\left(\int_{\Omega}|\nabla \bar{u}|^{2} d x\right) \int_{\Omega} \nabla \bar{u} \cdot \nabla \varphi d x & =A\left(\int_{\Omega}|\nabla \bar{u}|^{2} d x\right) \cdot \lambda \frac{C}{R} \int_{\Omega} \nabla \phi_{0} \cdot \nabla \varphi d x \\
& =\lambda \frac{C}{R} A\left(\int_{\Omega}|\nabla \bar{u}|^{2} d x\right) \int_{\Omega} \varphi d x \\
& \geq \lambda a_{1} \frac{C}{R} \int_{\Omega} \varphi d x
\end{aligned}
$$


Using the condition $\left(H_{3}\right)$, we can choose a number $C>0$ large enough so that

$$
C \lambda \geq \frac{\lambda R}{a_{1}} f\left(\frac{R \lambda}{b_{1}} g(C \lambda)\right)
$$

and therefore

$$
\begin{aligned}
A\left(\int_{\Omega}|\nabla \bar{u}|^{2} d x\right) \int_{\Omega} \nabla \bar{u} \cdot \nabla \varphi d x & \geq \lambda a_{1} \frac{C}{R} \int_{\Omega} \varphi d x \\
& \geq \lambda \int_{\Omega} f\left(\frac{R \lambda}{b_{1}} g(C \lambda)\right) \varphi d x \\
& \geq \lambda \int_{\Omega} f(\bar{v}) \varphi d x
\end{aligned}
$$

Next, from the definition of $\bar{v}$, the condition $\left(H_{1}\right)$ and the fact that $g$ is increasing, we also deduce that

$$
\begin{aligned}
B\left(\int_{\Omega}|\nabla \bar{v}|^{2} d x\right) \int_{\Omega} \nabla \bar{v} \cdot \nabla \varphi d x & =B\left(\int_{\Omega}|\nabla \bar{v}|^{2} d x\right) \frac{\lambda}{b_{1}} g(C \lambda) \int_{\Omega} \nabla \phi_{0} \cdot \nabla \varphi d x \\
& =B\left(\int_{\Omega}|\nabla \bar{v}|^{2} d x\right) \frac{\lambda}{b_{1}} g(C \lambda) \int_{\Omega} \varphi d x \\
& \geq \lambda g(C \lambda) \int_{\Omega} \varphi d x \\
& \geq \lambda \int_{\Omega} g\left(\frac{C \lambda}{R} \phi_{0}\right) \varphi d x \\
& =\lambda \int_{\Omega} g(\bar{u}) \varphi d x
\end{aligned}
$$

From (2.7) and (2.8), $(\bar{u}, \bar{v})$ is a supersolution of problem (1.1) with $\underline{u} \leq \bar{u}$ and $\underline{v} \leq \bar{v}$ for $C>0$ large.

In order to obtain a weak solution of problem (1.1) we shall use the arguments by Azzouz and Bensedik [2] (observe that $f, g$ does not depend on $x)$. For this purpose, we define a sequence $\left\{\left(u_{n}, v_{n}\right)\right\} \subset H_{0}^{1}\left(\Omega, \boldsymbol{R}^{2}\right)$ as follows: $u_{0}:=\bar{u}, v_{0}:=\bar{v}$ and $\left(u_{n}, v_{n}\right)$ is the unique solution of the system

$$
\left\{\begin{array}{l}
-A\left(\int_{\Omega}\left|\nabla u_{n}\right|^{2} d x\right) \Delta u_{n}=\lambda f\left(v_{n-1}\right) \quad \text { in } \Omega \\
-B\left(\int_{\Omega}\left|\nabla v_{n}\right|^{2} d x\right) \Delta v_{n}=\lambda g\left(u_{n-1}\right) \quad \text { in } \Omega \\
u_{n}=v_{n}=0, \quad \text { on } \partial \Omega .
\end{array}\right.
$$


System (2.9) is $(A, B)$-linear in the sense that, if $\left(u_{n-1}, v_{n-1}\right) \in H_{0}^{1}\left(\Omega, \boldsymbol{R}^{2}\right)$ is given, the right hand sides of (2.9) is independent of $u_{n}, v_{n}$. Set $\mathscr{A}(t)=t A\left(t^{2}\right)$, $\mathscr{B}(t)=t B\left(t^{2}\right)$. Then since $\mathscr{A}(\boldsymbol{R})=\boldsymbol{R}, \quad \mathscr{B}(\boldsymbol{R})=\boldsymbol{R}, \quad$ and $f\left(v_{n-1}\right) \in L^{2}(\Omega)$, $g\left(u_{n-1}\right) \in L^{2}(\Omega)$ (in $x$ ), we deduce from a result in [1] that system (2.9) has a unique solution $\left(u_{n}, v_{n}\right) \in H_{0}^{1}\left(\Omega, \boldsymbol{R}^{2}\right)$.

Using (2.9) and the fact that $\left(u_{0}, v_{0}\right)$ is a supersolution of (1.1) we have

$$
\begin{gathered}
-A\left(\int_{\Omega}\left|\nabla u_{0}\right|^{2} d x\right) \Delta u_{0} \geq \lambda f\left(v_{0}\right)=-A\left(\int_{\Omega}\left|\nabla u_{1}\right|^{2} d x\right) \Delta u_{1}, \\
-B\left(\int_{\Omega}\left|\nabla v_{0}\right|^{2} d x\right) \Delta v_{0} \geq \lambda g\left(u_{0}\right)=-B\left(\int_{\Omega}\left|\nabla v_{1}\right|^{2} d x\right) \Delta v_{1}
\end{gathered}
$$

and by Theorem 1.1, $u_{0} \geq u_{1}$ and $v_{0} \geq v_{1}$. Also, since $u_{0} \geq \underline{u}, v_{0} \geq \underline{v}$ and the monotonicity of $f, g$ one has

$$
\begin{aligned}
& -A\left(\int_{\Omega}\left|\nabla u_{1}\right|^{2} d x\right) \Delta u_{1}=\lambda f\left(v_{0}\right) \geq \lambda f(\underline{v}) \geq-A\left(\int_{\Omega}|\nabla \underline{u}|^{2} d x\right) \Delta \underline{u}, \\
& -B\left(\int_{\Omega}\left|\nabla v_{1}\right|^{2} d x\right) \Delta v_{1}=\lambda g\left(u_{0}\right) \geq \lambda g(\underline{u}) \geq-B\left(\int_{\Omega}|\nabla \underline{v}|^{2} d x\right) \Delta \underline{v}
\end{aligned}
$$

from which, according to Theorem $1.1, u_{1} \geq \underline{u}, v_{1} \geq \underline{v}$. For $u_{2}, v_{2}$ we write

$$
\begin{aligned}
& -A\left(\int_{\Omega}\left|\nabla u_{1}\right|^{2} d x\right) \Delta u_{1}=\lambda f\left(v_{0}\right) \geq \lambda f\left(v_{1}\right)=-A\left(\int_{\Omega}\left|\nabla u_{2}\right|^{2} d x\right) \Delta u_{2}, \\
& -B\left(\int_{\Omega}\left|\nabla v_{1}\right|^{2} d x\right) \Delta v_{1}=\lambda g\left(u_{0}\right) \geq \lambda g\left(u_{1}\right)=-B\left(\int_{\Omega}\left|\nabla v_{2}\right|^{2} d x\right) \Delta v_{2},
\end{aligned}
$$

and then $u_{1} \geq u_{2}, v_{1} \geq v_{2}$. Similarly, $u_{2} \geq \underline{u}$ and $v_{2} \geq \underline{v}$ because

$$
\begin{aligned}
& -A\left(\int_{\Omega}\left|\nabla u_{2}\right|^{2} d x\right) \Delta u_{2}=\lambda f\left(v_{1}\right) \geq \lambda f(\underline{v}) \geq-A\left(\int_{\Omega}|\nabla \underline{u}|^{2} d x\right) \Delta \underline{u}, \\
& -B\left(\int_{\Omega}\left|\nabla v_{2}\right|^{2} d x\right) \Delta v_{2}=\lambda g\left(u_{1}\right) \geq \lambda g(\underline{u}) \geq-B\left(\int_{\Omega}|\nabla \underline{v}|^{2} d x\right) \Delta \underline{v} .
\end{aligned}
$$

Repeating this argument we get a bounded monotone sequence $\left\{\left(u_{n}, v_{n}\right)\right\} \subset$ $H_{0}^{1}\left(\Omega, \boldsymbol{R}^{2}\right)$ satisfying

$$
\begin{gathered}
\bar{u}=u_{0} \geq u_{1} \geq u_{2} \geq \cdots \geq u_{n} \geq \cdots \geq \underline{u}>0, \\
\bar{v}=v_{0} \geq v_{1} \geq v_{2} \geq \cdots \geq v_{n} \geq \cdots \geq \underline{v}>0 .
\end{gathered}
$$

Using the continuity of the functions $f, g$ and the definition of the sequences $\left\{u_{n}\right\},\left\{v_{n}\right\}$, there exist two constant $C_{1}, C_{2}>0$ independent of $n$ 
such that

$$
\left|f\left(v_{n-1}\right)\right| \leq C_{1}, \quad\left|g\left(u_{n-1}\right)\right| \leq C_{2} \quad \text { for all } n .
$$

From (2.12), multiplying the first equation of (2.9) by $u_{n}$, integrating, using the Hölder inequality and the Sobolev embedding we can show that

$$
\begin{aligned}
a_{1} \int_{\Omega}\left|\nabla u_{n}\right|^{2} d x & \leq A\left(\int_{\Omega}\left|\nabla u_{n}\right|^{2} d x\right) \int_{\Omega}\left|\nabla u_{n}\right|^{2} d x \\
& =\lambda \int_{\Omega} f\left(v_{n-1}\right) u_{n} d x \\
& \leq \lambda \int_{\Omega}\left|f\left(v_{n-1}\right)\right|\left|u_{n}\right| d x \\
& \leq \lambda C_{1} \int_{\Omega}\left|u_{n}\right| d x \\
& \leq C_{3}\left\|u_{n}\right\|,
\end{aligned}
$$

or

$$
\left\|u_{n}\right\| \leq C_{3}, \quad \forall n
$$

where $C_{3}>0$ is a constant independent of $n$. Similarly, there exists $C_{4}>0$ independent of $n$ such that

$$
\left\|v_{n}\right\| \leq C_{4}, \quad \forall n .
$$

From (2.13), (2.14) we infer that $\left\{\left(u_{n}, v_{n}\right)\right\}$ has a subsequence which weakly converges in $H_{0}^{1}\left(\Omega, \boldsymbol{R}^{2}\right)$ to a limit $(u, v)$ with the properties $u \geq \underline{u}>0$ and $v \geq \underline{v}>0$. Being monotone and also using a standard regularity argument, $\left\{\left(u_{n}, v_{n}\right)\right\}$ converges itself to $(u, v)$. Now, lettting $n \rightarrow \infty$ in (2.9), we deduce that $(u, v)$ is a positive solution of system (1.1). The proof of Theorem 2.1 is now completed.

\section{Non-existence result}

In this section, we are interested in the non-existence of a positive solution for system (1.1). We consider system (1.1) under the particular nonlinearities:

$$
f(t):=c_{1} t^{q}-d_{1}, \quad g(t):=c_{2} t^{r}-d_{2}, \quad t \in \boldsymbol{R}^{+},
$$

where $c_{i}, d_{i}>0, i=1,2$ and $q, r \in(0,1)$. It should be noticed that the above nonlinearities satisfy the conditions $\left(\mathrm{H}_{2}\right)$ and $\left(\mathrm{H}_{3}\right)$. Moreover, the functions $f$ and $g$ defined as in (3.1) can change sign and they are sublinear at infinity in the sense that $\lim _{t \rightarrow+\infty} f(t) / t=\lim _{t \rightarrow+\infty} g(t) / t=0$. For this reason, our situation 
is different from [2] even in the single equation case. Now, system (1.1) can be read as follows

$$
\left\{\begin{array}{l}
-A\left(\int_{\Omega}|\nabla u|^{2} d x\right) \Delta u=\lambda\left(c_{1} v^{q}-d_{1}\right) \quad \text { in } \Omega \\
-B\left(\int_{\Omega}|\nabla v|^{2} d x\right) \Delta v=\lambda\left(c_{2} u^{r}-d_{2}\right) \text { in } \Omega \\
u=v=0, \quad \text { on } \partial \Omega .
\end{array}\right.
$$

Using Theorem 2.1, system (3.2) has at least one positive solution for $\lambda$ large enough. We shall prove that system (3.2) has no positive solution for $\lambda$ small enough. More precisely, we have the following result.

Theorem 3.1. Asume that the condition $\left(H_{1}\right)$ holds. Then there exists a positive constant $\lambda_{*}$ such that for any $0<\lambda<\lambda_{*}$ system (3.2) has no positive weak solution.

Proof. Let $\lambda_{1}$ be the first eigenvalue of $\left(-\Delta, H_{0}^{1}(\Omega)\right)$ and $\phi_{1}$ the corresponding positive eigenfunction. If $\left(u_{\lambda}, v_{\lambda}\right) \in H_{0}^{1}\left(\Omega, \boldsymbol{R}^{2}\right)$ is a positive solution of system (1.1) then

$$
-\int_{\Omega} \phi_{1} \Delta u_{\lambda} d x=\lambda_{1} \int_{\Omega} u_{\lambda} \phi_{1} d x
$$

and

$$
-\int_{\Omega} \phi_{1} \Delta v_{\lambda} d x=\lambda_{1} \int_{\Omega} v_{\lambda} \phi_{1} d x
$$

Hence,

$$
\begin{aligned}
a_{1} \lambda_{1} \int_{\Omega} \phi_{1} u_{\lambda} d x & \leq \lambda_{1} A\left(\int_{\Omega}\left|\nabla u_{\lambda}\right|^{2} d x\right) \int_{\Omega} \phi_{1} u_{\lambda} d x \\
& =-A\left(\int_{\Omega}\left|\nabla u_{\lambda}\right|^{2} d x\right) \int_{\Omega} \phi_{1} \Delta u_{\lambda} d x \\
& =\lambda \int_{\Omega} f\left(v_{\lambda}\right) \phi_{1} d x \\
& =\lambda \int_{\Omega}\left(c_{1} v_{\lambda}^{q}-d_{1}\right) \phi_{1} d x
\end{aligned}
$$

or

$$
\int_{\Omega} \phi_{1}\left(\lambda c_{1} v_{\lambda}^{q}-a_{1} \lambda_{1} u_{\lambda}\right) d x \geq \lambda d_{1} \int_{\Omega} \phi_{1} d x
$$


Similarly, from (3.4), we deduce that

$$
\int_{\Omega} \phi_{1}\left(\lambda c_{2} u_{\lambda}^{r}-b_{1} \lambda_{1} v_{\lambda}\right) d x \geq \lambda d_{2} \int_{\Omega} \phi_{1} d x
$$

From (3.5) and (3.6), it follows that

$$
\int_{\Omega} \phi_{1}\left[\left(\lambda c_{2} u_{\lambda}^{r}-a_{1} \lambda_{1} u_{\lambda}\right)+\left(\lambda c_{1} v_{\lambda}^{q}-b_{1} \lambda_{1} v_{\lambda}\right)\right] d x \geq \lambda\left(d_{1}+d_{2}\right) \int_{\Omega} \phi_{1} d x
$$

Since $q, r \in(0,1)$ the maximums of the functions $u_{\lambda} \mapsto \lambda c_{2} u_{\lambda}^{r}-a_{1} \lambda_{1} u_{\lambda}$ and $v_{\lambda} \mapsto \lambda c_{1} v_{\lambda}^{q}-b_{1} \lambda_{1} v_{\lambda}$ are achieved at $u_{\lambda}=\left(a_{1} \lambda_{1} /\left(\lambda r c_{2}\right)\right)^{1 /(r-1)}$ and at $v_{\lambda}=$ $\left(b_{1} \lambda_{1} /\left(\lambda q c_{1}\right)\right)^{1 /(q-1)}$, respectively. Hence, by some simple calculations, it follows from (3.7) that

$$
\int_{\Omega} \phi_{1}\left[L_{1} \lambda^{1 /(1-r)}+L_{2} \lambda^{1 /(1-q)}\right] d x \geq \lambda\left(d_{1}+d_{2}\right) \int_{\Omega} \phi_{1} d x
$$

or

$$
L_{1} \lambda^{r /(1-r)}+L_{2} \lambda^{q /(1-q)} \geq d_{1}+d_{2}>0
$$

where $L_{i}, i=1,2$, are positive constants independent of $\lambda$. By (3.8), there exists a positive constant $\lambda_{*}$ such that for any $\lambda \in\left(0, \lambda_{*}\right)$, system (3.2) has no positive solution. The proof of Theorem 3.1 is now completed.

\section{References}

[ 1 ] Alves, C. O. and Corrêa, F. J. S. A., On existence of solutions for a class of problem involving a nonlinear operator, Comm. Appl. Nonlinear Anal., 8 (2001), 43-56.

[2 $]$ Azzouz, N. and Bensedik, A., Existence results for an elliptic equation of Kirchhoff-type with changing sign data, Funkcial. Ekvac., 55 (2012), 55-66.

[3] Chipot, M. and Lovat, B., Some remarks on nonlocal elliptic and parabolic problems, Nonlinear Anal., 30 (1997), 4619-4627.

[ 4 ] Corrêa, F. J. S. A. and Figueiredo, G. M., On an elliptic equation of $p$-Kirchhoff type via variational methods, Bull. Austral. Math. Soc., 74 (2006), 263-277.

[ 5 ] Corrêa, F. J. S. A. and Figueiredo, G. M., On a $p$-Kirchhoff equation via Krasnoselskii's genus, Appl. Math. Lett., 22 (2009), 819-822.

[6] Hai, D. D. and Shivaji, R., An existence result on positive solutions for a class of $p$-Laplacian systems, Nonlinear Anal., 56 (2004), 1007-1010.

[ 7 ] Han, X. and Dai, G., On the sub-supersolution method for $p(x)$-Kirchhoff type equations, J. Inequal. Appl., 2012: 283 (2012), 11pp.

[8] Kirchhoff, G., Mechanik, Teubner, Leipzig, Germany, 1883.

[9] Ma, T. F., Remarks on an elliptic equation of Kirchhoff type, Nonlinear Anal., 63 (2005), 1967-1977.

[10] Perera, K. and Zhang, Z., Nontrivial solutions of Kirchhoff-type problems via the Yang index, J. Differential Equations, 221 (2006), 246-255. 
[11] Ricceri, B., On an elliptic Kirchhoff-type problem depending on two parameters, J. Global Optim., 46 (2010), 543-549.

[12] Sun, J. J. and Tang, C. L., Existence and multiplicity of solutions for Kirchhoff type equations, Nonlinear Anal., 74 (2011), 1212-1222.

[13] Zhang, Z. and Perera, K., Sign changing solutions of Kirchhoff type problems via invariant sets of descent flow, J. Math. Anal. Appl., 317 (2006), 456-463.

\author{
nuna adreso: \\ G. A. Afrouzi \\ Department of Mathematics \\ Faculty of Mathematical Sciences \\ University of Mazandaran \\ Babolsar \\ Iran \\ E-mail: afrouzi@umz.ac.ir \\ N. T. Chung \\ Department of Mathematics \\ Quang Binh University \\ 312 Ly Thuong Kiet, Dong Hoi \\ Quang Binh \\ Vietnam \\ E-mail: ntchung82@yahoo.com \\ S. Shakeri \\ Department of Mathematics \\ Ayatollah Amoli Branch \\ Islamic Azad University \\ Amol \\ Iran \\ E-mail: s.shakeri@iauamol.ac.ir
}

(Ricevita la 12-an de januaro, 2014)

(Reviziita la 20-an de marto, 2015) 